\title{
Cerebellar degeneration with Hodgkin's disease
}

\author{
L. HORWICH, P. H. BUXTON, AND G. M. S. RYAN \\ From the Neuropathology Laboratory, Neurosurgical Centre, Walton Hospital, Liverpool
}

The association of neurological symptoms with Hodgkin's disease is uncommon when there is no direct involvement of the central nervous system by tumour tissue. This paper describes a patient in whom the first symptoms of neurological disorder appeared four months before the clinical features of Hodgkin's disease developed.

\section{CASE REPORT}

A male glassworker, aged 19, who had previously been in good health developed severe occipital headache, made worse by movements of the head and by stooping. A fortnight later the headache had become bitemporal and gradually less severe so that he was able to return to work. During this initial period no abnormal signs appeared in the nervous system or elsewhere although the blood pressure was somewhat labile and was variously recorded as $150 / 90,135 / 75$, and $140 / 100 \mathrm{~mm}$. $\mathrm{Hg}$.

Two months later he was referred again because of brief dizzy spells for some of which he had to be taken home from work. During these he would have to hold on to objects to avoid falling and there would at times be nausea and vomiting. A week later he improved sufficiently to attempt to drive a car to visit relatives but on the way he developed double vision and was forced to return. Some days later he drove a hundred miles without trouble. Whilst on holiday, however, double vision recurred, his gait became unsteady, and his speech indistinct.

Neurological examination 10 weeks from the onset revealed a pure cerebellar disorder with staccato speech, and a horizontal nystagmus to the left and right. There was such marked ataxia of the limbs and trunk that he was unable to stand unsupported. There was no weakness or sensory loss and the deep and superficial reflexes were normal with flexor plantar responses. The optic fundi were normal and the visual fields full. The remainder of the cranial nerves and the rest of the nervous system showed no abnormality apart from the cerebellar signs.

He was admitted to hospital for detailed investigation. The peripheral blood showed a haemoglobin level of $116 \%(16.9 \mathrm{~g}$.) white blood cells 8,400 per c.mm. (neutrophils $80 \%$, lymphocytes $18 \%$, monocytes $2 \%$ ). The E.S.R. was $14 \mathrm{~mm}$. in one hour. Urine examination was normal. The total serum protein concentration was $6.5 \mathrm{~g} . \%$ (albumin 3.5 , globulin $2 \cdot 7$, fibrinogen 0.3 ).

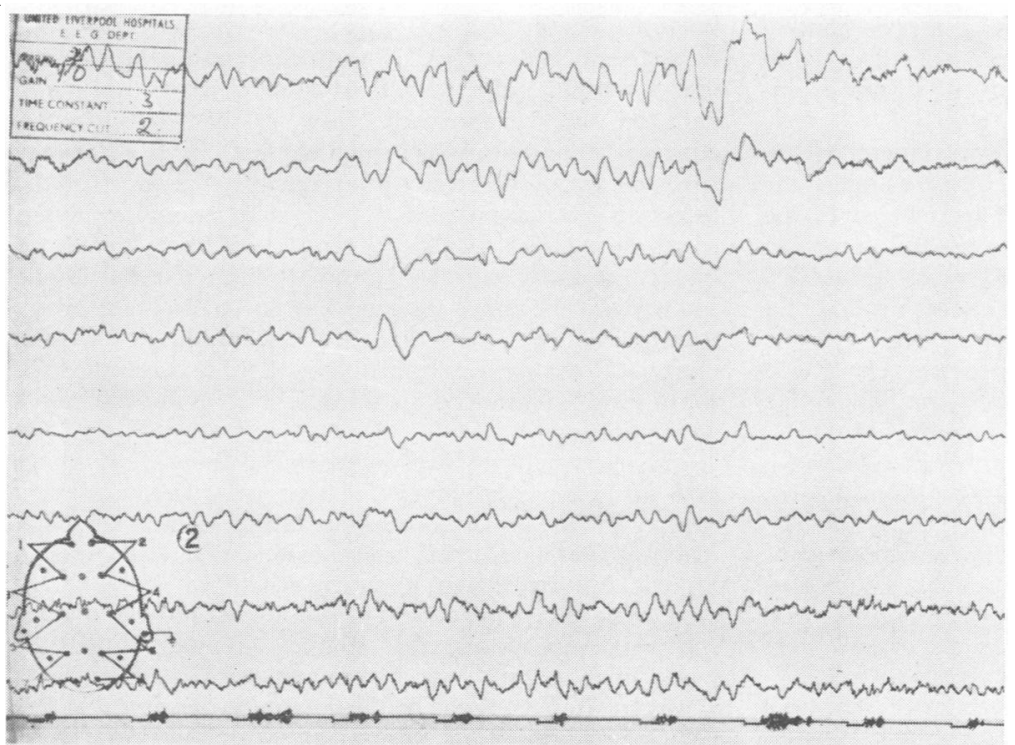

FIG. 1. Electroencephalogram showing excessive slow activity in all areas. 
The cerebrospinal fluid was clear and colourless, under a pressure of $135 \mathrm{~mm}$. with less than 1 cell per c.mm. The total protein content of the fluid was 60 mg. per $100 \mathrm{ml}$. , the globulin content was not increased, and the gold curve showed 0000000000 . The Wassermann reaction in both blood and cerebrospinal fluid was negative.

A chest radiograph on admission was normal. Bilateral carotid and vertebral angiograms were also normal.

The E.E.G. showed gross abnormalities. Very little alpha activity was present, the basic rhythms being predominantly delta and theta which were equal over both hemispheres (Fig. 1). The slow activity was appreciably increased by hyperventilation.

On completing the preliminary investigation, A.C.T.H. (20) units twice daily) was started as a symptomatic measure and was continued for eight weeks. During this period the patient became slightly less unsteady and was able to walk three paces between hospital beds unaided.

Two weeks after completing the course of A.C.T.H. he developed a fever of $100^{\circ}$ to $104^{\circ} \mathrm{F}$. Routine blood cultures were sterile and there was no response to tetracycline. The white cell count at this time rose steadily to 20,000/ c.mm., the differential count being polymorphs $87 \%$, lymphocytes $3 \%$, and eosinophils $10 \%$. For the first time the lower cervical lymph nodes became enlarged and a second chest radiograph showed the superior mediastinal shadow to be widened by enlarged lymph nodes. The sternal marrow was extremely cellular due to marked myeloid activity but the myeloid series was morphologically normal and erythropoiesis was normoblastic. A Paul-Bunnell test was positive to a titre of only 1 in 32 and the dye dilution and complement-fixation tests for toxoplasmosis were negative.

A cervical node was removed and showed Hodgkin's disease. Cyclophosphamide, $100 \mathrm{mg}$. twice daily, was then administered for four and a half weeks during which time the white cell count was reduced to $5,000 / \mathrm{c} . \mathrm{mm}$., but there was no improvement in the patient's general condition. The lymph nodes continued to enlarge and fever persisted. As the head hair began to fall out cyclophosphamide was discontinued.

During this time there was no improvement in the cerebellar dysfunction; speech remained slurred and there was some weakness of the palate and disturbance of the gag reflex. In the next four months the general condition gradually deteriorated although the neurological disorder remained essentially unchanged and at no time did consciousness or intellect become impaired.

Ten months after the onset the patient developed respiratory distress and high fever once more. The white cell count rose to $42,000 / \mathrm{c} . \mathrm{mm}$. (polymorphs $96 \%$, lymphocytes $4 \%$ ). The chest radiograph now showed a large mass in the superior mediastinum on the right side extending far out into the right lung field.

On examination, nystagmus and other cerebellar signs persisted as did the weakness of the gag reflex. The triceps tendon reflexes were present, but the biceps, knee, and ankle reflexes could not now be obtained. There were still no long tract abnormalities.

The patient died a week after the onset of this final episode.
POST-MORTEM FINDINGS A fluctuant subcutaneous swel-Z ling present over the manubrium was continuous with aC necrotic mediastinal mass, 5 in. in diameter, which hadô infiltrated the wall of the trachea and the sternum, ande had indented the upper lobe of the right lung. There was bilateral bronchopneumonia but no extensive consolidation, and no macroscopical direct involvement of lung by tumour.

The pericardium was thickened beneath the medias tinal mass, but the heart appeared normal. The liver. showed congestion with necrotic nodules up to $2 \mathrm{~cm} \Rightarrow$

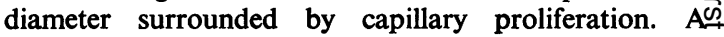
necrotic mass was present in the region of the porta hepatis, but no biliary obstruction. The spleen $(400 \mathrm{~g}$. $)$ ? was slightly enlarged, of a homogeneous fleshy, pink colour, and showed loss of normal structure. The kidneyso had a streaky appearance due to pyelonephritis. Lymph nodes in the neck and mesentery were enlarged and firmo

Externally and on macroscopical section the brain $\overrightarrow{5}$ showed slight atrophy of the cerebellum and olives, but no gross abnormality. No tumour tissue was seentu within the skull.

MICROSCOPY Sections of mediastinal mass, liver nodules, and spleen all showed infiltration by reticulum cells multinucleate giant cells, and large numbers of eosino:phils. Bands of fibrous tissue were prominent and thetes was much necrosis (Figs. 2 and 3). The appearances we्gre? typical of Hodgkin's disease.

Central nervous system The stains used on parafinembedded material were haematoxylin and eosin, Nised cresyl violet, Heidenhein stain for myelin, phosphotungs tic acid haematoxylin, and Holzer's crystal violet of glial fibres; on frozen sections, oil red $\mathrm{O}$, periodic aside Schiff, buffered toluidine blue, Bielchowsky's sil w̄èr hydroxide method for axis cylinders, and Cajal's gäh sublimate method for astrocytes were employed.

The most striking changes were present in the cere? bellum. There was extensive loss of Purkinje cells throughout, but particularly in the vermis (Fig. 4); the few remaining cells, seen in small groups in the laterah part of the hemispheres, showed shrinkage, hypero chromasia, and homogenizing change. In Bielchowsky silver preparations basket fibres surrounded some of the Purkinje cell remnants and occasional axonal swellings were seen (Fig. 6). Some tangential fibres remainede intact but many were short and stunted. The Bergmann? astrocytes were much increased, even in the areas where Purkinje cells persisted, and there was a mild, diffuse glia increase in the molecular cell layer (Fig. 5). Granule cells were generally reduced in number but abnormal forms were not seen.

The nerve cells of the dentate nuclei showed shrinkage and homogenizing change. There was diffuse loss op myelin in the surrounding area, which contained manyo macrophages filled with sudanophilic droplets (Fig. 7)? Similar diffuse myelin loss was found in some of the cerebellar folia, particularly those more centrally placed In these demyelinated areas, and also in some of the folid where myelin loss was slight, marked fibrillary gliosis was seen (Fig. 8). Some of these astrocytes were large but bizarre, multinucleate, tumour-like forms were not seen 


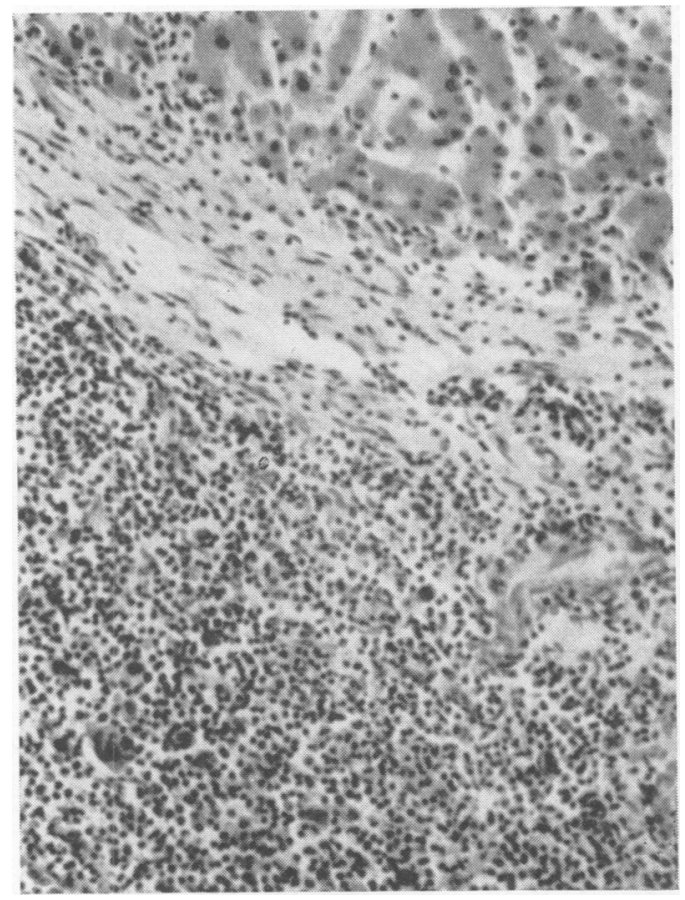

FIG. 2. Section of tumour nodule in liver. Haematoxylin and $\operatorname{eosin} \times 160$.

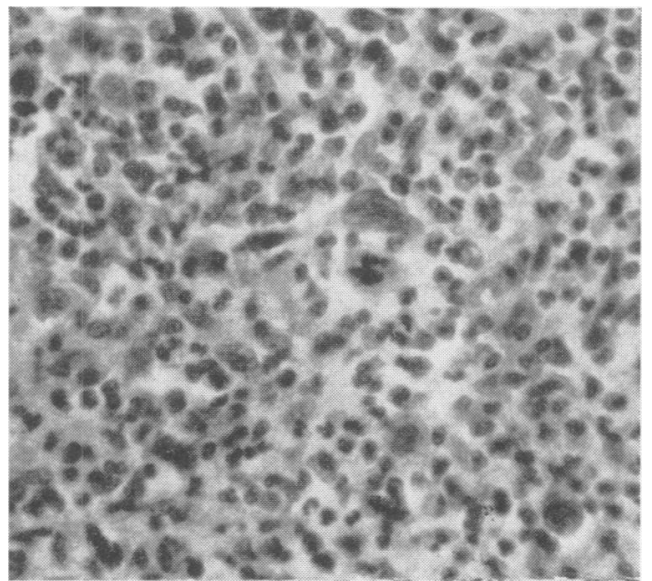

FIG. 3. Section of mediastinal tumour showing reticulum cells and tumour giant cells. Haematoxylin and eosin $\times$ 350 .

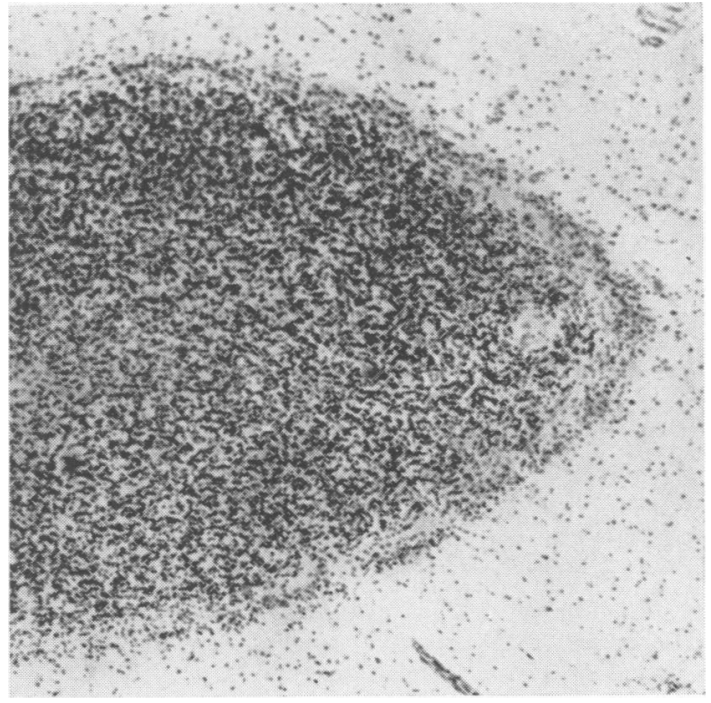

FIG. 4. Cerebellar folium showing absence of Purkinje cells and increase in Bergman astrocytes. Nissl $\times 65$.

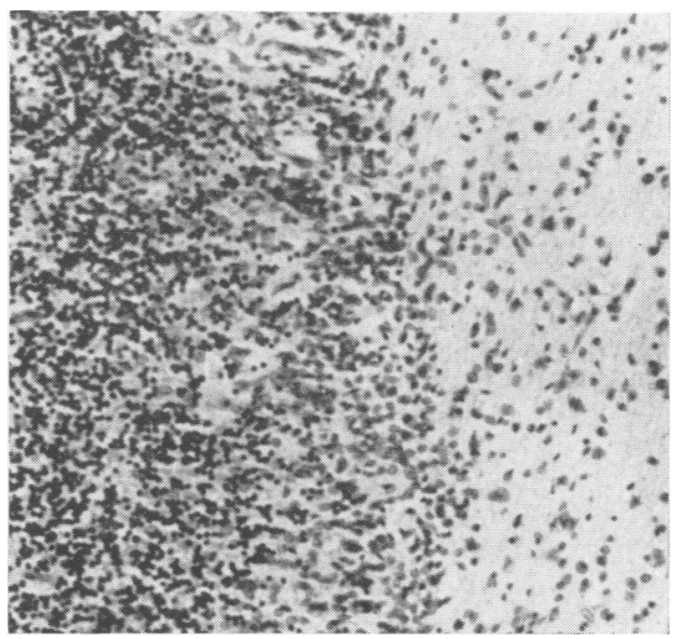

FIG. 5. Same cerebellar folium as in Fig. 4. Nissl $\times 350$. 


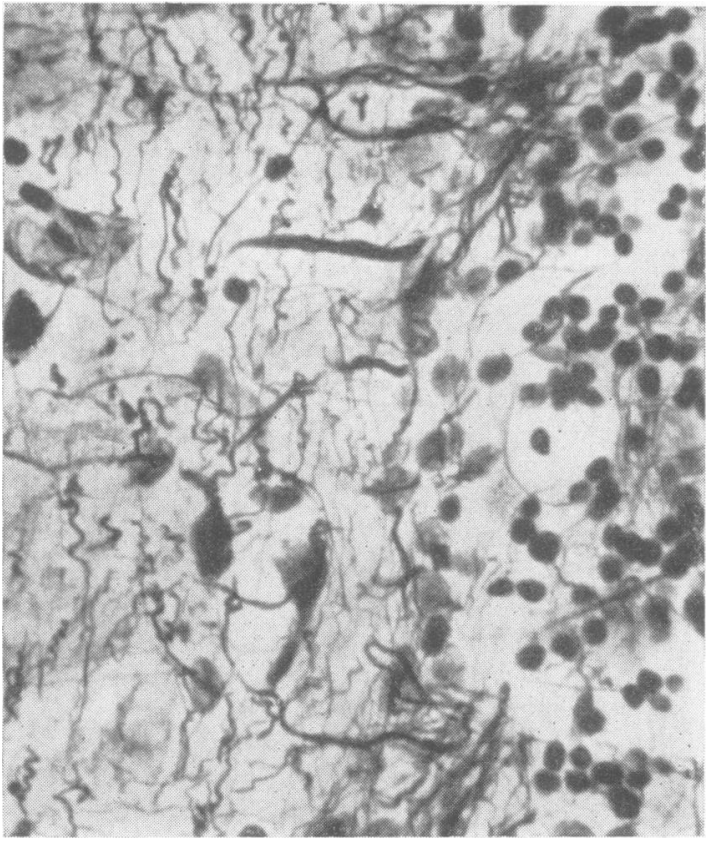

FIG. 6. Cerebellar cortex showing degenerate Purkinje cell remnants and one swollen axon. Tangential fibres are short and irregular. Bielchowsky $\times 650$.

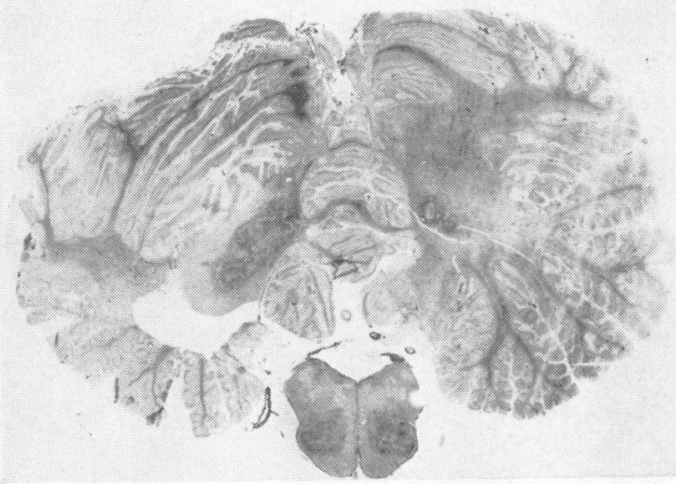

FIG. 8. Cerebellum and medulla, showing gliosis around dentate and olivary nuclei and in white matter of cerebellar folia. Holzer $\times 1+$.

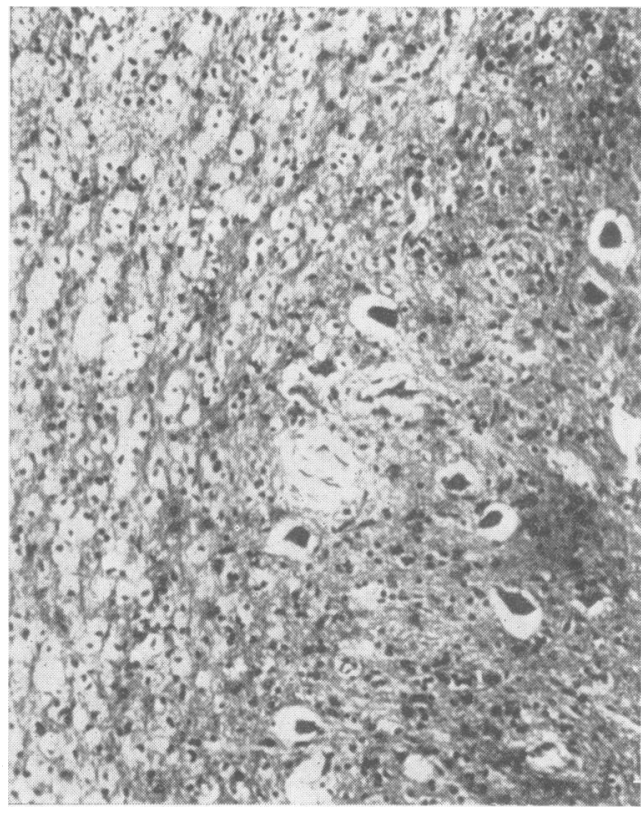

FIG. 7. Dentate nucleus showing shrinkage of nerve ce and lipid macrophages in adjacent white matter. Heidesthain's myelin $\times 350$.

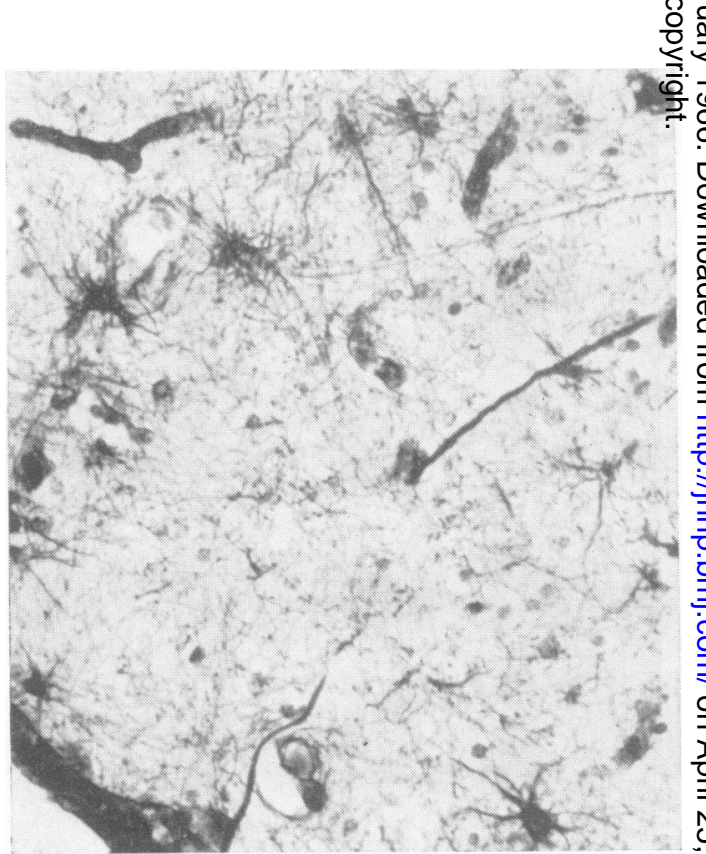

FIG. 9. Edge of olive showing large fibrillary astrocytes. $N$ Holzer $\times 350$

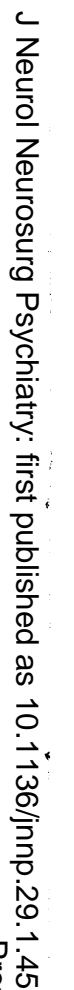

फ

(1)

$\sum$

ֻัญ

은

훙

言. 
Abnormal enlarged oligodendrocytes and eosinophilic inclusions were not found.

The inferior cerebellar peduncles showed mild myelin loss and gliosis but no definite changes were seen in the superior and middle peduncles and in the nuclei pontis. There was glial increase, including a few large astrocytes in the inferior olives, particularly in the hilar region, and although some apparently viable nerve cells persisted, many were shrunken, distorted, and hyperchromatic (Fig. 9). Occasional degenerate nerve cells were seen also in the vagal and hypoglossal nuclei.

No gross changes were found in the red nuclei, substantia nigra, or subthalamic nuclei; nor in the thalamus, mammillary bodies, putamen, or globus pallidus. Sections of frontal, temporal, parietal, and occipital cortex were examined and no significant lesions were found, apart from mild nerve cell loss and moderate gliosis in Ammon's horn, which may well have been due to terminal hypoxia associated with pulmonary infection.

Perivascular lymphocytic cuffing was noted around some brain-stem and cerebellar capillaries and arterioles, and occasionally in the cerebral hemispheres where it was most apparent in the subcortical white matter of the temporal lobes. A few clusters of lymphocytes were seen in relation to the meninges, but there was no significant infiltration.

The whole spinal cord was not available but in the upper cervical region no spino-cerebellar or other tract degeneration could be shown by oil red $\mathrm{O}$ or myelin stains.

\section{DISCUSSION}

Attention was drawn to the relation between subacute cortical cerebellar degeneration and carcinoma by Brain, Daniel, and Greenfield (1951). They reviewed previously reported cases of subacute cortical cerebellar degeneration and added four cases of their own. In these cases cerebellar symptoms developed fairly rapidly, progressing from unsteadiness of gait to include clumsiness of the hands and dysarthric speech, till in a few weeks or months the patient was no longer able to sit up unsupported. In the whole group of 16 cases, 11 were associated with carcinoma. This association in the subacute cases contrasted with chronic forms of cerebellar degeneration where no such association exists. Henson, Russell, and Wilkinson (1954) reported 19 cases of carcinomatous neuropathy and myopathy; five of them had cerebellar symptoms, but only one showed marked cerebellar cortical degeneration, though degeneration of the dentate nucleus, olives, subthalamic nuclei, and motor neurones of the spinal cord and medulla oblongata was found in another. Brain and Henson (1958) reported further cases and pointed out that neurological symptoms could antedate the clinical manifestations or diagnosis of the carcinoma by at least three years. These reports of non-metastatic neurological complications of malignant disease have all been in association with carcinoma.

In 1958, Aström, Mancall, and Richardson reported three cases of unusual demyelinating lesions; in one the patient had died of Hodgkin's disease, in the other two cases of leukaemia. Subsequent similar reports have appeared by Cavanagh, Greenbaum, Marshall, and Rubinstein (1959) and Lloyd and Urich (1959). A comprehensive report by Richardson (1961) summarized 22 previously recorded cases of progressive multifocal leucoencephalopathy and gave a detailed description of the clinical features and pathology. This condition usually occurs against a background of chronic disease affecting the reticuloendothelial system, including the reticuloses and leukaemias, carcinomatosis, sarcoidosis, and miliary tuberculosis. The clinical neurological manifestations are diffuse, eventually bilateral but asymmetrical, and whilst the cerebral hemispheres are always affected, lesions may occur in the brain-stem, cerebellum, and basal ganglia.

Brain (1963) reviewed the neurological complications of neoplasms and discussed the non-metastatic complications in three main groups: encephalomyeloneuropathy, including subacute cerebellar cortical degeneration associated with carcinoma; myopathic-myasthenic syndrome; and progressive multifocal leucoencephalopathy associated with chronic disease of the reticulo-endothelial system, most often Hodgkin's disease, leukaemia, or lymphosarcoma.

The only previous report of a case of subacute cerebellar degeneration associated with Hodgkin's disease is that of Rewcastle (1963) and he could only find one similar case recorded, that included by Malamud in his atlas of neuropathology (1957). Rewcastle's case occurred in a 62-year-old man, but, apart from the age of the patient, the clinical and pathological features are strikingly similar to the present case. In both cases initial symptoms of headache, dizziness, and diplopia were followed after a short remission by progressive cerebellar symptoms. Constituents of the cerebrospinal fluid were normal in Rewcastle's case and an E.E.G. showed only a minimal irregularity over the left frontal area. Another finding common to both cases was the leucocytosis with eosinophilia $(18,300$ per c.mm. with $7 \%$ eosinophilia in Rewcastle's case, and 20,000 per c.mm. with $10 \%$ eosinophilia in our case). In both cases these high eosinophil counts were made about five months after the initial symptoms, at the time the Hodgkin's disease became apparent; in our case the white cell count had been normal on the first admission. Rewcastle found a reduction in size of enlarged lymph nodes and a 
partial remission in the cerebellar symptoms after a course of mechlorethamine, whereas the present case failed to respond to cyclophosphamide, a similar cytotoxic agent.

Histologically also both cases were very similar and showed much in common with the cases of subacute cerebellar degeneration associated with carcinoma, such as depletion of Purkinje cells, axonal swellings, moderate loss of granule cells, demyelination, and gliosis of dentate nuclei and similar less intense changes in the cerebellar folia.

Focal demyelination was not seen in the cerebral hemispheres in the present case. Other typical features of multifocal leucoencephalopathy as mentioned by Richardson (1961), such as abnormal oligodendrocytes, eosinophilic inclusions, abnormal granule cells, bizarre or tumorose astrocytes, were not found.

Hutchinson, Leonard, Maudsley, and Yates (1958) reviewed 229 patients with reticulosis and found 45 with neurological disorders. These included an unusual form of myelomalacia, peripheral neuropathy, and papilloedema without raised intracranial pressure. Haynal and Regli (1964) reported 27 patients who presented with a lesion of the nervous system out of an unspecified total of cases of Hodgkin's disease treated in the university neurological clinic at Zurich. In these cases the cerebral lesions were the result of focal demyelination and included cerebellar symptoms and also a granulomatous angiitis going on to meningoencephalitis. Cranial and peripheral nerve lesions as well as paraplegia were discussed and one case of polymyositis in Hodgkin's disease was described. Deep, Fraumeni, Tashima, and McDivitt (1964) report leucoencephalopathy and dermato-myositis in a case of Hodgkin's disease. In none of these papers were there any case reports of subacute cerebellar cortical degeneration associated with reticulosis.

The aetiology of non-metastatic neurological disorders complicating malignant disease remains obscure. If there is a general aetiological explanation, it must account for the rarity of these conditions, and the fact that more than one type of neurological disorder may complicate the same type of malignancy, possibly even in one individual. Furthermore the neurological disorder may precede or follow the appearance of malignancy, undergo a spontaneous remission, be arrested by surgery or chemotherapy directed at the malignant disease, and may even appear for the first time after such treatment. The same type of neurological disease, particularly multifocal leucoencephalopathy, may complicate benign conditions or even appear in elderly but otherwise normal subjects.

In the present case there was no known industrial or hereditary predisposition to neurological or $\bar{Z}$ malignant disease. The subacute stuttering onset $\stackrel{\Phi}{C}$ suggested the start of an inflammatory or vascular process, but at this time there was no pyrexia, and the blood count was normal. Carotid and vertebral ${ }^{6}$ angiograms were also normal. The cerebrospinal on fluid was not examined till two months after the clinical onset but then showed only a slight rise in $\frac{0}{0}$. protein content. At necropsy no inclusions were $\bar{z}$ found and perivascular cuffing, though nowhere $\Rightarrow$ intense, was more evident in the cerebellum and $\stackrel{\vec{\rho}}{?}$ brain-stem where it was thought to be reactive to neuronal degeneration rather than the result of $\frac{\overline{ }}{\bar{N}}$ infection.

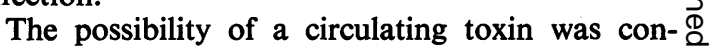
sidered, particularly in view of the high eosinophil \% count noted also in Rewcastle's case, which occurred at the time of the enlargement of glands due to Hodgkin's disease, and the known relationship $\vec{\omega}$ between Purkinje cell loss and experimental injection of eosinophils. However, considerable damage to $\overline{\overline{3}}$ the cerebellum clearly took place four months before the enlargement of lymph glands at a time when there 0 was no increase in circulating eosinophils. The E.E.G $\overrightarrow{0}$ abnormalities were an unexpected finding in cerê. bellar degeneration, although Liversedge and Emef (1961) have described similar changes where the was no rise in intracranial pressure. It was thoug that the abnormalities in the present case arose im 음 the cerebral hemispheres although they were ngi the changes usually found in association with Ammon's horn sclerosis. Possibly they were eviden $\overrightarrow{0}$ of some toxin to which the cerebral cortical nerve $\sigma$ cells were more resistant than the cerebellar neurones.

An alternative possibility that the agent producing the neurological damage also promoted the Hodgkin's disease seems unlikely, in view of similar cases where cortical cerebellar degeneration antedates $\frac{2}{D}$ carcinoma arising in various organs.

In general, neoplastic-neuropathic associations follow patterns as described by Brain (1963), but $\exists$ less common associations may be important in providing aetiological clues although so far they only appear to have eliminated a type-specific concept.

\section{SUMMARY}

A case of subacute cerebellar degeneration with Hodgkin's disease is described. The subject was a 을 19-year-old man who presented with intermittent $>$ headache and vertigo. A severe cerebellar disorder followed, becoming only slowly progressive in the later stages. Five months after the onset of neuro- $\mathrm{N}$ logical symptoms enlarged supraclavicular glands $N$ appeared. A biopsy of a cervical lymph node showed స్心 
Hodgkin's tissue. The condition was treated first with A.C.T.H. and later with cyclophosphamide without material benefit. Death occurred 10 months after the onset.

At necropsy Hodgkin's disease of the mediastinal lymph nodes with tumour nodules in the porta hepatis and liver was confirmed. The brain showed cerebellar cortical degeneration with patchy demyelination and gliosis. Degeneration and gliosis were also prominent in the dentate nuclei and olives. Less severe nerve cell loss and gliosis occurred in Ammon's horn.

We thank Dr. F. S. Mooney, St. Helen's Hospital, for the initial biopsy report.

\section{REFERENCES}

Aström, K. E., Mancall, E. L., and Richardson, E. P. Jr. (1958). Progressive multifocal leuko-encephalopathy: a hitherto unrecognized complication of chronic lymphatic leukaemia and Hodgkin's disease. Brain, 81, 93-111.

Brain, W. R. (1963). The neurological complications of neoplasms. Lancet, 1, 179-184.
- Daniel, P. M., and Greenfield, J. G. (1951). Subacute cortical cerebellar degeneration and its relation to carcinoma. $J$. Neurol. Neurosurg. Psychiat., 14, 59-75.

- , and Henson, R. A. (1958). Neurological syndromes associated with carcinoma. The carcinomatous neuromyopathies. Lancet, 2, 971-974.

Cavanagh, J. B., Greenbaum, D., Marshall, A. H., and Rubinstein, L. J. (1959). Cerebral demyelination associated with disorders of the reticuloendothelial system. Ibid., 2, 524-529.

Deep, W. D., Fraumeni, J. F., Tashima, C. K., and McDivitt, R. (1964). Leukoencephalopathy and dermatomyositis in Hodgkin's disease. Arch. intern. Med., 113, 635-640.

Haynal, A., and Regli, F. (1964). Neurologische, Symptome bei Morbus Hodgkin. Schweiz. med. Wschr., 94, 1515-1518.

Henson, R. A., Russell, D. S., and Wilkinson, M. (1954). Carcinomatous neuropathy and myopathy. A clinical and pathological study. Brain, 77, 82-121.

Hutchinson, E. C., Leonard, B. J., Maudsley, C., and Yates, P. O. (1958). Neurological complications of the reticuloses. Ibid., 81, 75-92.

Liversedge, L. A., and Emery, V. (1961). Electroencephalographic changes in cerebellar degenerative lesions. J. Neurol. Neurosurg. Psychiat., 24, 326-330.

Lloyd, O. C., and Urich, H. (1959). Acute disseminated demyelination of the brain associated with lymphosarcoma. Lancet, 2, $529-530$.

Malamud, N. (1957). Atlas of Neuropathology. University of California Press, Berkeley.

Rewcastle, N. B. (1963). Subacute cerebellar degeneration with Hodgkin's disease. Arch. Neurol. (Chic.), 9, 407-413.

Richardson, E. P. Jr. (1961). Progressive multifocal leukoencephalopathy. New Engl. J. Med., 265, 815-823. 